\title{
Regulation of mPGES-1 composition and cell growth via the MAPK signaling pathway in jurkat cells
}

\author{
YI-QING LI ${ }^{1,2^{*}}$, JIAO-TING CHEN ${ }^{1-3^{*}}$, SONG-MEI YIN ${ }^{1}$, DA-NIAN NIE ${ }^{1}$, ZHI-YUAN HE $^{1}$, \\ SHUANG-FENG XIE ${ }^{1}$, XIU-JU WANG ${ }^{1}$, YU-DAN WU ${ }^{1}$, JIE XIAO ${ }^{1}$, HONG-YUN LIU ${ }^{1}$, \\ JIE-YU WANG ${ }^{1}$, WEN-JUAN YANG ${ }^{1}$ and LI-PING MA ${ }^{1}$ \\ ${ }^{1}$ Department of Hematology, Sun Yat-Sen Memorial Hospital, Sun Yat-Sen University; \\ ${ }^{2}$ Guangdong Provincial Key Laboratory of Malignant Tumor Epigenetic and Gene Regulation, \\ Sun Yat-Sen Memorial Hospital, Sun Yat-Sen University, Guangzhou, Guangdong 510120; ${ }^{3}$ Department of Hematology, \\ The Sixth Affiliated Hospital, Sun Yat-Sen University, Guangzhou, Guangdong 510655, P.R. China
}

Received January 10, 2018; Accepted June 22, 2018

DOI: $10.3892 /$ etm.2018.6538

\begin{abstract}
Previous studies have suggested that microsomal prostaglandin E synthase-1 (mPGES-1) is highly expressed and closely associated with mitogen-activated protein kinase (MAPK) signaling pathways in various types of malignant cells. However, their expression patterns and function with respect to T-cell acute lymphoblastic leukemia (T-ALL) remain largely unknown. The present study investigated whether mPGES-1 served a crucial role in T-ALL and aimed to identify interactions between mPGES-1 and the MAPK signaling pathway in T-ALL. The results indicated that mPGES-1 overexpression in T-ALL jurkat cells was significantly decreased by RNA silencing. Decreasing mPGES-1 on a consistent basis may inhibit cell proliferation, induce apoptosis and arrest the cell cycle in T-ALL jurkat cells. Microarray and western blot analyses revealed that c-Jun N-terminal kinase served a role in the mPGES-1/prostaglandin E2/EP4/MAPK positive feedback loops. In addition, P38 and extracellular signal-regulated kinase $1 / 2$ exhibited negative feedback effects on mPGES-1. In conclusion, the results suggested that cross-talk between mPGES-1 and the MAPK signaling pathway was very complex. Therefore, the combined regulation of mPGES-1 and the MAPK signaling pathway may be developed into a new candidate therapy for T-ALL in the future.
\end{abstract}

Correspondence to: Professor Song-Mei Yin or Professor Da-Nian Nie, Department of Hematology, Sun Yat-Sen Memorial Hospital, Sun Yat-Sen University, 107 Yanjiang Xi Road, Guangzhou, Guangdong 510120, P.R. China

E-mail: sumyinsongmei@163.com

E-mail: niedanian@hotmail.com

*Contributed equally

Key words: feedback, jurkat cell, microsomal prostaglandin-E synthase, mitogen activated protein Kinase Signaling System, T-cell acute lymphoblastic leukemia

\section{Introduction}

T-cell acute lymphoblastic leukemia (T-ALL) is an aggressive hematological malignancy, which accounts for $25 \%$ of adult ALL cases (1). Although the clinical outcome has been dramatically improved by a combination of chemotherapy and hematopoietic stem cell transplantation, the prognosis of T-ALL remains poor due to a high frequency of induction failure and early relapse. Therefore, continued studies to identify innovative modes of action in T-ALL and the development of specific targeting therapies are still urgently required (2).

The effect of prostaglandin E2 (PGE2) on cell growth has attracted attention in recent years. Previous studies have demonstrated that the secretion of PGE2 in endometrial cancer, colon cancer, colorectal cancer, ovarian cancer and other malignant cells is significantly increased $(3,4)$, indicating that PGE2 is implicated in the occurrence and progression of cancer. Microsomal prostaglandin E synthase-1 (mPGES-1) is the terminal synthase responsible for converting COX-derived PGH2 into PGE2 (5). Non-steroidal anti-inflammatory drugs (NSAIDs) may reduce the synthesis of PGE2 by inhibiting COX and affecting various biological functions of tumors (6-8). However, due to the gastrointestinal and cardiovascular side effects of NSAIDs, their clinical application has been limited $(9,10)$. In recent years, overexpression of mPGES-1 was observed in a number of solid tumors (11-13). A preliminary study by the authors confirmed for the first time that mPGES-1 is highly expressed in human acute myeloid leukemia (AML) primary cells and AML cell lines such as HL-60. Inhibiting mPGES-1/PGE2 may induce apoptosis, inhibit proliferation, arrest the cell cycle and improve chemosensitivity (14-16); however, the roles of mPGES-1/PGE2 in T-ALL cells are largely unknown.

Mitogen-activated protein kinases (MAPKs), including extracellular signal-regulated kinase (ERK1/2), c-Jun N-terminal kinase (JNK) and P38 subtypes, are a highly-conserved family of serine/threonine kinases that serve an important role in regulating cell growth, differentiation, inflammatory reactions and cancer progression $(17,18)$. Previous studies have revealed that different MAPKs may be involved 
in the regulation of mPGES-1 expression induced by inflammatory stimuli (19-21). Interestingly, certain previous studies have hypothesized that the MAPK signaling pathway resides upstream of PGE2 and regulates the synthesis of PGE2 through early growth response protein-1 (EGR-1) (19), while in other contexts, it is located downstream of mPGES-1/PGE2 $(20,21)$. The MAPK signaling pathway, once activated, may be further regulated by complex feedback loops exerting either positive or negative effects on cascade components (22). The present study aimed to investigate the effects of mPGES-1 on T-ALL jurkat cells in vitro and attempted to determine the interaction between mPGES-1 and MAPKs in jurkat cells.

\section{Materials and methods}

Materials. Human T-ALL jurkat cell line was obtained from the Hematology Research Institute (Tianjin, China). The EP4 receptor antagonist L-161982 was purchased from Cayman Chemical Company (Ann Arbor, MI, USA). The MEK1/2 inhibitor U0126, JNK inhibitor SP600125 and the P38 inhibitor SB203580 were purchased from Selleck Chemicals (Shanghai, China). The anti-ERK1/2 (cat. no. 9926; dilution, 1:1,000), anti-p-ERK1/2 (Thr202/Tyr204; cat. no. 9910; dilution, 1:2,000), anti-P38 (cat. no. 9926; dilution, 1:1,000), anti-p-P38 (Thr180/Tyr182; cat. no. 9910; dilution, 1:1,000), anti-JNK (cat. no. 9926; dilution, 1:1,000), anti-p-JNK (Thr183/Tyr185; cat. no. 9910; dilution, 1:1,000), anti-EGR-1 (cat. no. 4153; dilution, 1:1,000), anti-GAPDH (cat. no. 2118; dilution, 1:1,000) and anti-rabbit $\mathrm{IgG}$, horseradish peroxidase-conjugated (cat. no. 7074; dilution, 1:2,000) antibodies were purchased from Cell Signaling Technology, Inc. (Danvers, MA, USA) and anti-mPGES-1 antibody (cat. no. 10004350; dilution, 1:1,000) was purchased from the Cayman Chemical Company.

Cell culture. Jurkat cells were cultured in RPMI 1640 medium containing $10 \%$ fetal bovine serum (both Gibco; Thermo Fisher Scientific, Inc., Waltham, MA, USA) at $37^{\circ} \mathrm{C}$ in $5 \% \mathrm{CO}_{2}$. To test the effect of EP4 receptor, jurkat cells (8x10 $/$ well) were plated in 6-well plate and incubated with antagonist L-161982 (10 mM stock solution in dimethyl sulfoxide, further dissolved with RPMI 1640 to give a $33.3 \mu \mathrm{M}$ working solution) for $24 \mathrm{~h}$ at $37^{\circ} \mathrm{C}$ in $5 \% \mathrm{CO}_{2}$.

Lentivirus infection. Gene knockdown was performed using lentivirus short hairpin RNA (shRNA), which was synthesized by GeneChem Co., Ltd., (Shanghai, China). The shRNA was cloned into pLKO.1 (GV115) lentiviral vectors (hU6-MCS-CMV-EGFP, GeneChem Co., Ltd., Shanghai, China) at $40 \mathrm{nmol} / 1$. Four shRNA-mPGES-1 targeting sequences (27740-1, 5'-GGGCTTCGTCTACTC CTTT-3'; 27741-1, 5'-TGCTGGTCATCAAGATGTA-3'; 27742-1, 5'-GGCTAAGAATGCAGACTTT-3' and 27743-1, 5'-TTTCTGGTCCCTTCAGTAT-3') were designed. The shRNA-negative control (NC) targeting sequence was 5'-TTC TCCGAACGTGTCACGT-3'. The culture containing lentivirus was added to the jurkat cells in the presence of $5 \mu \mathrm{g} / \mathrm{ml}$ polybrene (Shanghai GeneChem Co., Ltd.). Positively transfected cells were selected by $1 \mu \mathrm{g} / \mathrm{ml}$ puromycin after $24 \mathrm{~h}$ incubation at $37^{\circ} \mathrm{C}$ in $5 \% \mathrm{CO}_{2}$. Stable cell lines were verified by western blot analysis as described below.
Cell proliferation assay. Cell proliferation was measured using a Cell Counting kit-8 (Dojindo Molecular Technologies, Inc., Kumamoto, Japan) in vitro. A total of $1 \times 10^{4}$ cells were plated per well in 96 -well plates and incubated at $37^{\circ} \mathrm{C}$ with $5 \% \mathrm{CO}_{2}$ for 24,48 and $72 \mathrm{~h}$. The cells were divided into three groups: i) KD group, jurkat cells transfected with shRNA (27743-1) targeting mPGES-1; ii) NC group, jurkat cells transfected with NC shRNA; and iii) Control (CON) group, jurkat cells without any treatment. A total of $10 \mu \mathrm{l}$ CCK- 8 was added to each well and the samples were incubated for a further $4 \mathrm{~h}$. The optical density (OD) values were measured using a microplate reader (Bio-Rad Laboratories, Inc., Hercules, CA, USA) at $450 \mathrm{~nm}$.

Flow cytometry. Following incubation in a serum-free RPMI 1640 medium overnight, jurkat cells were collected (115 x g, $5 \mathrm{~min}$, room temperature) and rinsed twice with PBS. For cell cycle analysis, a total of $5 \times 10^{5}$ cells were fixed with $70 \%$ pre-chilled ethanol overnight at $4^{\circ} \mathrm{C}$ and stained with propidium iodide for $10 \mathrm{~min}$ at room temperature. The DNA content was analyzed by a BD FACStar flow cytometer (BD Biosciences, Franklin Lakes, NJ, USA). A total of $1 \times 10^{6}$ cells were washed and re-suspended in binding buffer and subsequently incubated with $5 \mathrm{ml}$ Annexin V-fluorescein isothiocyanate (PE Annexin V Apoptosis Detection kit 1; BD Biosciences) for $15 \mathrm{~min}$ at room temperature in the dark. A total of $2.5 \mathrm{ml}$ allophycocyanin (BD Biosciences) was added and the cells were analyzed with a FACScan flow cytometer (FACSCalibur; BD Biosciences). Fluorophores were excited at $640 \mathrm{~nm}$. Data acquisition and analysis were performed using CellQuest software (v6.1x; BD Biosciences).

GeneChip assay. GeneChip assays were performed by Shanghai GeneChem Co., Ltd. (Shanghai, China). General steps were as follows: Total RNA was extracted using TRIzol reagent (Takara Bio, Inc., Otsu, Japan) according to the manufacturer protocol. The quantity and quality of the RNA were determined by spectrophotometer and $1 \%$ formaldehyde denaturing gel electrophoresis. An Affymetrix Gene Chip ${ }^{\circledR}$ Prime View ${ }^{\mathrm{TM}}$ Human Gene Expression array was used for the microarray analysis. Hybridization, data capture and analysis were performed by Shanghai GeneChem Co., Ltd. (Shanghai, China). Briefly, $100 \mathrm{ng}$ total RNA was used for cDNA synthesis and biotin-tagged cRNA was produced by the Gene Chip IVT Labeling kit (Affymetrix; Thermo Fisher Scientific, Inc.). A total of $15 \mu \mathrm{g}$ fragmented cRNA, with the controls oligo B2 and eukaryotic hybridization, were hybridized to each GeneChip array at $45^{\circ} \mathrm{C}$ for $16 \mathrm{~h}$ (Affymetrix Gene Chip Hybridization Oven 640) according to the manufacturer protocol. Following hybridization, the Gene Chip arrays were washed three times at room temperature and stained with streptavidin phycoerythrin onan $\left(3 \mathrm{x} ; 35^{\circ} \mathrm{C} ; 300 \mathrm{sec}\right)$ with Affymetrix Fluidics Station 450 followed by scanning with the Affymetrix Gene Chip Scanner 30007G. Molecular function and signaling pathways were analyzed using Gene Ontology (GO; http://www.geneontology. org/) and Kyoto Encyclopedia of Genes and Genomes (KEGG; https://www.kegg.jp/kegg/kegg4.html), respectively.

Western blot analysis. Cells were lysed with an appropriate volume of the radioimmunoprecipitation buffer (CWBIO; Biotechnology Co., Ltd., Beijing, China) 
Table I. Gene expression values in the MAPK signaling pathway following mPGES-1 silencing.

\begin{tabular}{lccc}
\hline & \multicolumn{2}{c}{ Treatment group } & \\
\cline { 2 - 3 } Gene & $\mathrm{KD}$ & $\mathrm{NC}$ & $\begin{array}{c}\text { Direction of } \\
\text { regulation }\end{array}$ \\
\hline ARRB2 & $-0.329 \pm 0.059$ & $0.328 \pm 0.062$ & Down \\
FOS & $-0.276 \pm 0.039$ & $0.280 \pm 0.025$ & Down \\
PPP3CB & $-0.449 \pm 0.035$ & $0.424 \pm 0.050$ & Down \\
FGFR3 & $-0.447 \pm 0.080$ & $0.429 \pm 0.059$ & Down \\
MAP3K8 & $-0.320 \pm 0.072$ & $0.327 \pm 0.146$ & Down \\
ZAK & $-0.156 \pm 0.061$ & $0.164 \pm 0.089$ & Down \\
CACNA1A & $0.279 \pm 0.032$ & $-0.324 \pm 0.074$ & Up \\
PRKACB & $0.476 \pm 0.079$ & $-0.491 \pm 0.092$ & Up \\
IL1R2 & $0.442 \pm 0.089$ & $-0.404 \pm 0.067$ & Up \\
RAC1 & $0.381 \pm 0.061$ & $-0.407 \pm 0.027$ & Up \\
DUSP2 & $0.298 \pm 0.017$ & $-0.300 \pm 0.014$ & Up \\
NR4A1 & $0.239 \pm 0.029$ & $-0.298 \pm 0.028$ & Up \\
\hline
\end{tabular}

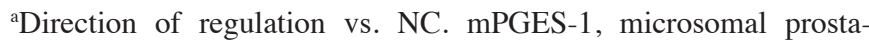
glandin E synthase-1; MAPK, mitogen-activated protein kinase; NC, negative control; KD, jurkat cells transfected with shRNA (27743-1).
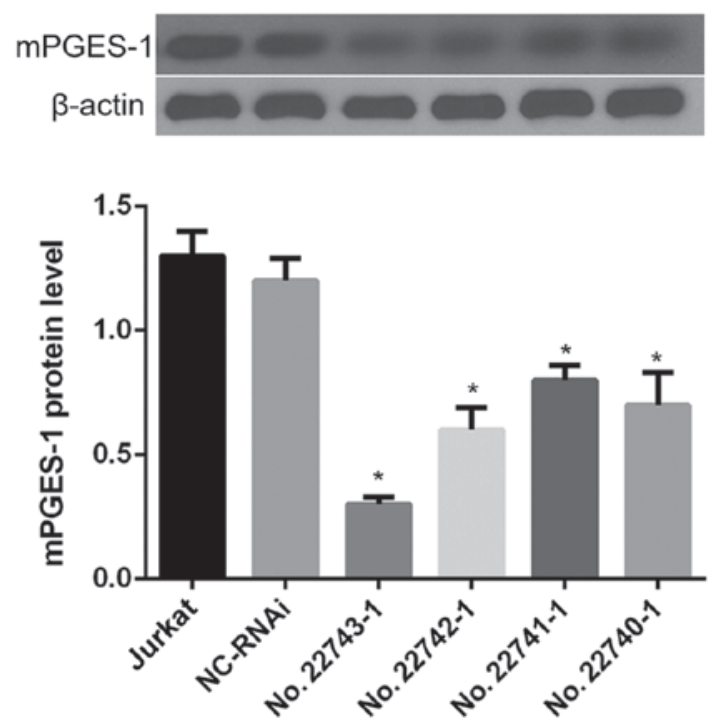

Figure 1. Lentiviral interference efficiency. Western blot analysis of the mPGES-1 protein expression in jurkat cells following lentiviral infection ${ }^{*} \mathrm{P}<0.05$ vs. jurkat group. mPGES-1, microsomal prostaglandin E synthase- 1 NC, negative control; mPGES-1, microsomal prostaglandin E synthase-1.

supplemented with protease inhibitor cocktail (CWBIO; Biotechnology Co., Ltd., Beijing, China) and the protein concentrations were determined by bicinchoninic acid assays with bovine serum albumin (BSA; CWBIO, Biotechnology Co., Ltd., Beijing, China) as the standard. A total of $30 \mathrm{ng} / 20 \mu \mathrm{l}$ protein was separated by $10 \%$ SDS-PAGE and transferred to polyvinylidene difluoride membranes (EMD Millipore, Billerica, MA, USA). Following blocking with Tris-buffered saline (TBS) containing 5\% BSA diluted in TBS with Tween-20 for $1 \mathrm{~h}$, the membranes were incubated overnight at $4^{\circ} \mathrm{C}$ with primary antibodies diluted according to the instruction followed by incubation with the horseradish peroxidase-conjugated secondary antibodies for $1 \mathrm{~h}$ at room temperature. The immunoreactive bands were detected using a chemiluminescent system (Thermo Fisher Scientific, Inc.) and quantified using ImageJ 1.43 (National Institutes of Health, Bethesda, MD, USA).

Statistical analysis. All experiments were performed three times. Data were processed using SPSS 20.0 software (IBM Corp., Armonk, NY, USA) and presented as the mean \pm standard deviation. Statistical analysis was conducted using one-way analysis of variance followed by Student-Newman-Keuls post-hoc tests. $\mathrm{P}<0.05$ was considered to indicate a statistically significant difference.

\section{Results}

The results of gene silencing by $R N A$ interference. To establish a useful cell line, the expression of mPGES-1 was decreased via lentivirus shRNA interference. Following western blot analysis of the results, sequence 27743-1 was selected for use in the following experiments (as the KD group) as it displayed the highest interference rate when transfected into jurkat cells $(\mathrm{P}<0.05$; Fig. 1). An inverted fluorescence microscope was used to observe the cells; it revealed that the KD and $\mathrm{NC}$ groups had a similar morphology to the CON group (Fig. 2A), indicating that the lentivirus infection had no notable effects on cell morphology. Subsequently, flow cytometry was utilized to detect the infection efficiency. The results revealed that the infection efficiency of the KD and $\mathrm{NC}$ groups were 84.87 and $83.17 \%$, respectively $(\mathrm{P}<0.05$; Fig. $2 \mathrm{~B}$ ). Infection efficiency allowed for cells to be used in further experiment.

mPGES-1 silencing inhibits proliferation, induces apoptosis and arrests the cell cycle in jurkat cells. To evaluate the effect of mPGES-1 on the proliferation of jurkat cells, a CCK-8 experiment was conducted. The results revealed that the proliferation of the KD group was significantly slower compared with the NC and CON groups at 24, 48 and $72 \mathrm{~h}$ $(\mathrm{P}<0.05)$, whereas no significant difference was observed between the $\mathrm{NC}$ group and the CON group at any time point (Fig. 3). In addition, the percentage of total apoptotic cells (total Annexin-V-FITC+ cells) was significantly increased in the KD group compared with the $\mathrm{CON}$ and $\mathrm{NC}$ groups when assayed by flow cytometry ( $\mathrm{P}<0.05$; Fig. 4A). The populations of early (Annexin-V-FITC+, PI- cells) and late apoptotic cells (Annexin-V-FITC+,PI+ cells) were also significantly increased in the KD group compared with the NC and CON groups $(\mathrm{P}<0.05$; Fig. 4A). Silencing mPGES-1 may also influence the cell cycle of jurkat cells. The percentage of cells in the G1 phase was significantly increased, while the percentage at the $\mathrm{S}$ phase was significantly reduced in the KD group compared with the $\mathrm{NC}$ and $\mathrm{CON}$ groups $(\mathrm{P}<0.05$; Fig. 4B). These results indicated that decreasing mPGES-1 inhibited proliferation, induced apoptosis and arrested the cell cycle the G1 phase in jurkat cells.

Reducing the expression of $M P G E S-1$ inhibits the MAPK signaling pathway. To further understand the mechanism of mPGES-1's effects on jurkat cells, microarray analysis was 
A

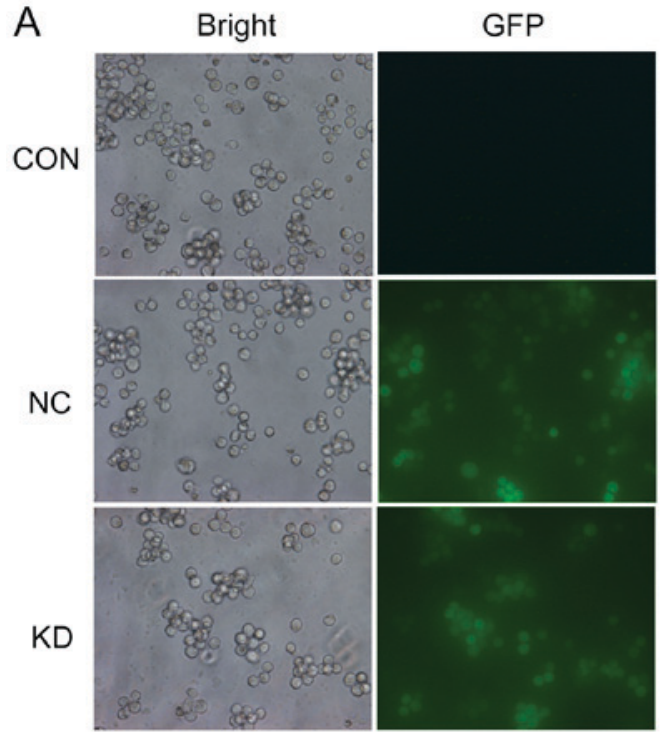

B

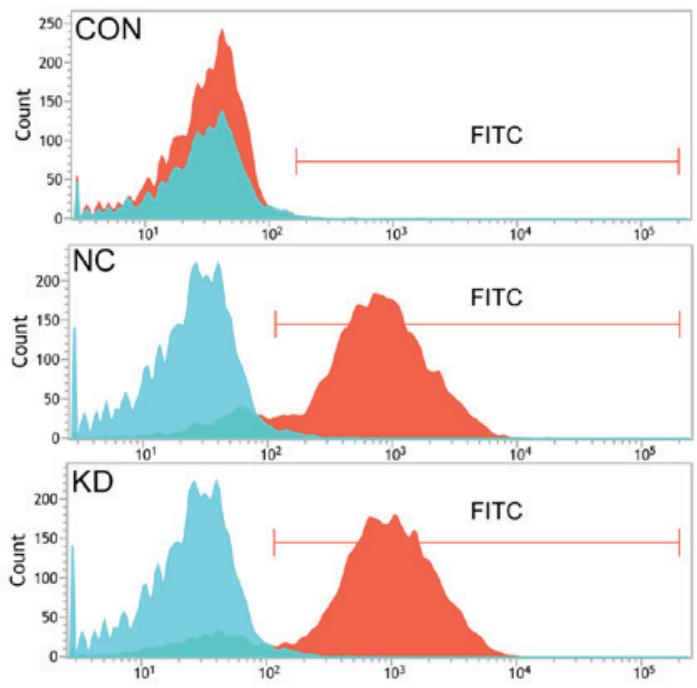

Figure 2. Lentiviral infection efficiency. (A) Representative images of jurkat cells following lentiviral infection. (B) Flow cytometry analysis of the lentiviral infection efficiency of jurkat cells. NC, negative control; GFP, green fluorescent protein; CON, control; KD, jurkat cells transfected with shRNA (27743-1).

used to detect the changes in gene expression following knockdown of mPGS-1. It was revealed that 456 genes had changed significantly following the knockdown of mPGES-1. These genes primarily participated in cell proliferation, apoptosis, protein metabolism and immune response as indicated by GO analysis (data not shown). The associated signaling pathways were analyzed via KEGG software and the results indicated that MAPK had the clearest change in all relevant signaling pathways. The MAPK signaling pathway was significantly inhibited $\left(\mathrm{P}=6.78 \times 10^{-4}\right)$ following knockdown of mPGES-1 (Fig. 5A). A total of 12 genes in the MAPK signaling pathway, including ARRB2, FOS, PPP3CB, FGFR3, MAP3K8, ZAK, CACNA1A, PRKACB, IL1R2, RAC1, DUSP2 and NR4A1 were involved (Fig. 5B and Table I). These findings revealed that the biological function of jurkat cells may be associated with the MAPK signaling pathway, and that mPGES-1 may be located upstream of it.

The MAPK signaling pathway is one of the most important signal transduction systems. It participates in cell growth, development, differentiation, and other physiological and pathological processes (23). Previous studies have demonstrated that the major MAPK signaling pathway subfamilies associated with COX-2/mPGES-1 or lipopolysaccharide-activated inflammatory responses may be ERK1/2, JNK and P38 (23). There is cross-talk between these three components, which leads to either coordination or inhibition (24). To identify which signaling pathway is associated with the function of mPGES-1 in jurkat cells, the phosphorylation status of major MAPK subfamilies was investigated following mPGES-1 silencing. The expression levels of phosphorylated (p)-P38 and p-ERK1/2 were significantly increased, while p-JNK was significantly decreased compared with the NC group $(\mathrm{P}<0.05$; Fig. 6). Based on these results, the authors speculated that mPGES-1 may affect the growth of jurkat cells through the JNK/MAPK signaling pathway. Alternatively, decreasing mPGES-1 may activate the P38 MAPK and ERK1/2/MAPK signaling pathways.

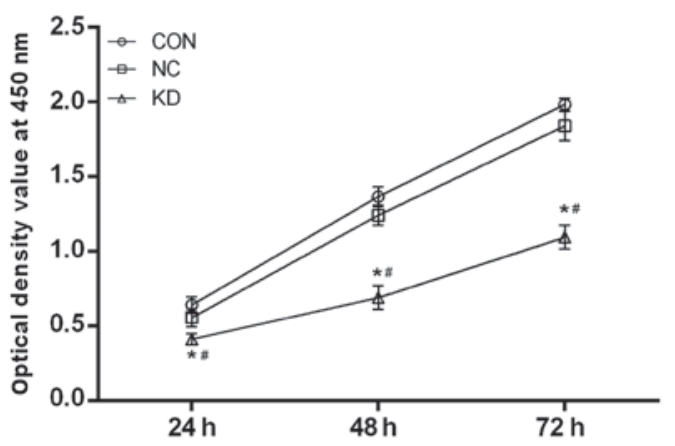

Figure 3. Effect of mPGES-1 silencing on the proliferation of jurkat cells The proliferation of jurkat cells was observed at 24,48 and $72 \mathrm{~h}$ following lentiviral infection; this was measured by a cell counting kit- 8 assay. ${ }^{*} \mathrm{P}<0.05$ vs. $\mathrm{CON}$; ${ }^{\text {P }}<0.05$ vs. NC. mPGES-1, microsomal prostaglandin E synthase-1; CON, control; KD, jurkat cells transfected with shRNA (27743-1); NC, negative control.

Whether the subfamilies are regulated independently or as a result of cross-talk is a question, which requires further investigation.

EP4 receptor mediates the regulation of $M P G E S-1$ on the $M A P K$ signaling pathway. PGE2 has diverse actions and stimulates key downstream signal transduction pathways by binding to its prostanoid receptors (EP1, EP2, EP3 and EP4) (25). Binding of PGE2 to different receptors may lead to the activation of different signaling pathways (25). A previous study by the authors reported that mPGES-1/PGE2 was closely associated with MAPKs, however, which subtype of prostanoid receptors mediated the activation of MAPKs remained unknown. In the present study jurkat cells were pre-incubated with EP4 receptor antagonist L-161982 and then the phosphorylation of MAPKs was examined. It was observed that the changes in the MAPKs were consistent with the results obtained following silencing of mPGES-1 $(\mathrm{P}<0.05$; Fig. 7). This may indicate that $\mathrm{mPGES}-1 / \mathrm{PGE} 2$ regulates MAPKs by combining with the EP4 receptor. 

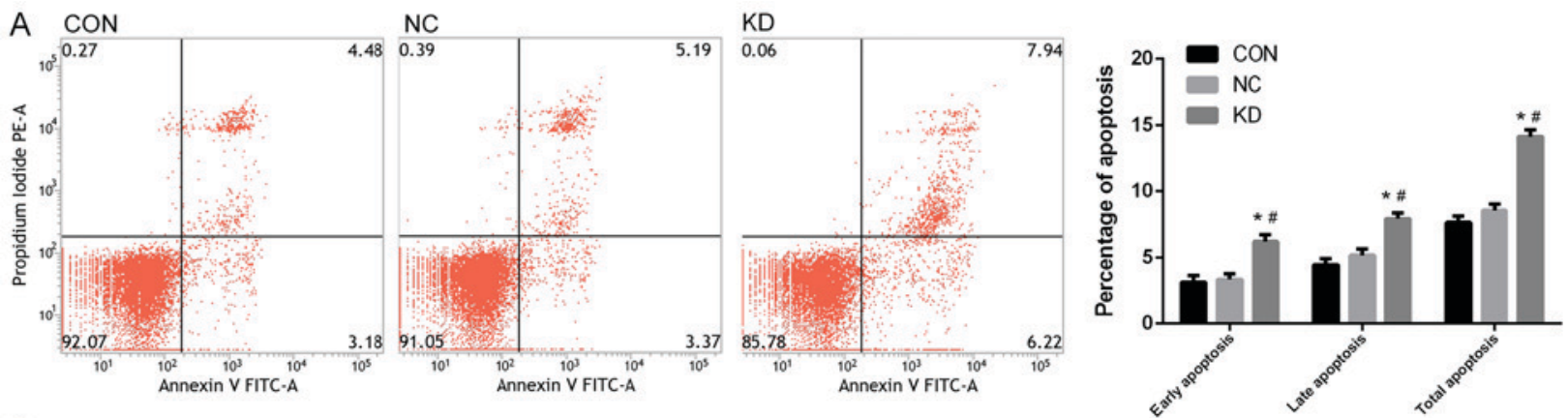

B
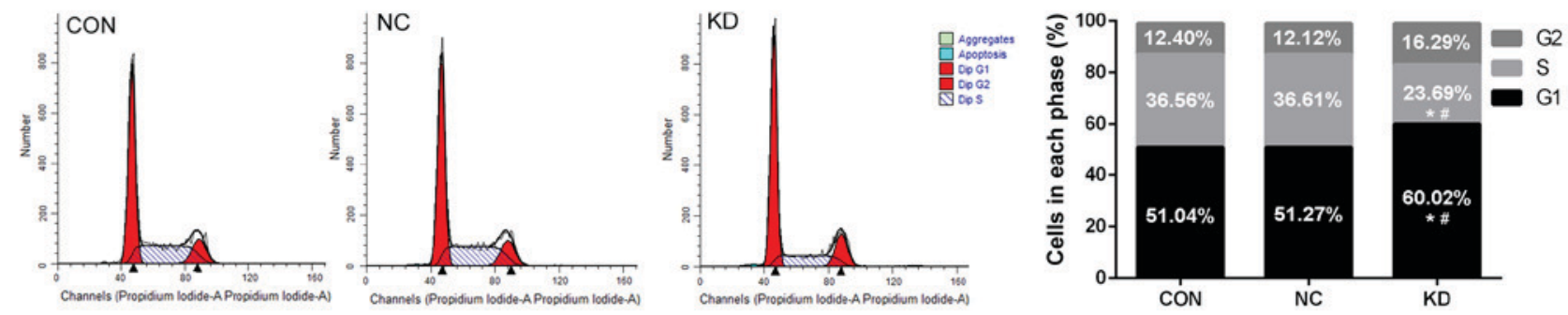

Figure 4. Effect of mPGES-1 silencing on the apoptosis and cell cycle of jurkat cells. (A) Cell apoptosis and (B) cell cycle distribution in jurkat cells following lentiviral infection, as determined by flow cytometry. "P<0.05 vs. CON; " $\mathrm{P}<0.05$ vs. NC. mPGES-1, microsomal prostaglandin E synthase-1; CON, control; KD, jurkat cells transfected with shRNA (27743-1); NC, negative control; FITC, fluorescein isothiocyanate.

A

En positive $z$-score $\square z$-score $=0$ negative $z$-score $\mid$ no activity pattern avalable $=-$ - Ratio

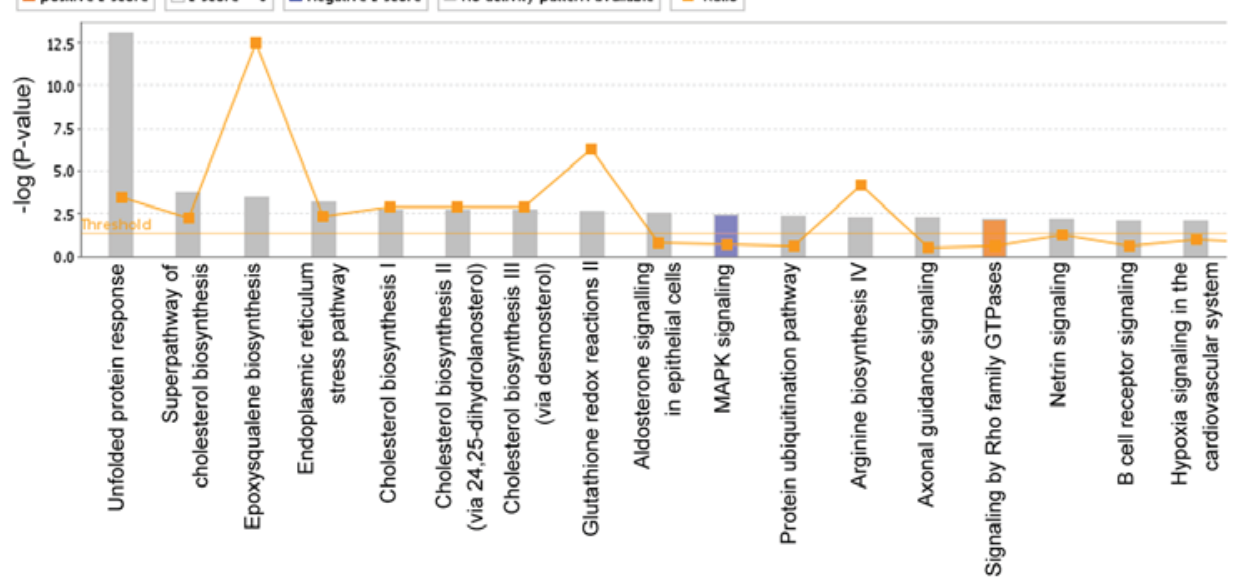

B $\quad-1.0$

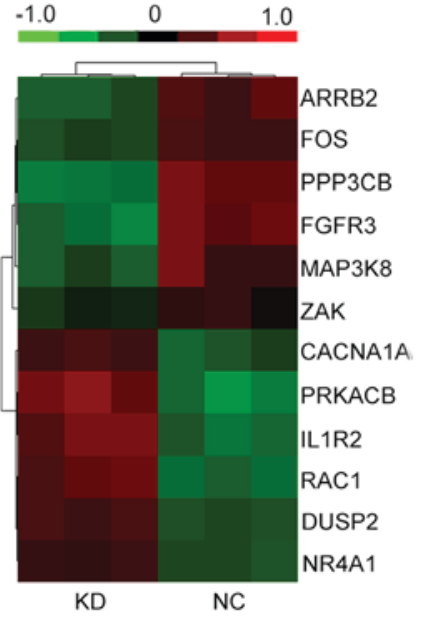

Figure 5. Effect of mPGES-1 silencing on signaling pathways. (A) Signaling pathway histogram revealed the enrichment of differential genes in classic pathways. Orange histogram represents a pathway, which is activated; blue histogram represents a pathway, which is inhibited. The ratio represents (number of genes changed significantly in the pathway)/(number of all genes in the pathway). (B) Heat map of gene expression values involved in the MAPK signaling pathway. mPGES-1, microsomal prostaglandin E synthase-1; MAPK, mitogen-activated protein kinase; KD, jurkat cells transfected with shRNA (27743-1); NC, negative control.

MAPKs feedback on mPGES-1 may be partly via EGR-1. Several previous studies seem to indicate that MAPKs regulate mPGES-1 via EGR-1, a transcription factor that regulates the composition of mPGES-1 (26-29). The authors hypothesized that MAPKs may regulate the expression of mPGES-1 by regulating EGR-1 in jurkat cells. To confirm this hypothesis, the jurkat cells were treated with inhibitors U0126, SB203580 and SP600125 against the phosphorylation of ERK1/2, P38 and JNK, respectively. It was revealed that following the inhibition of the ERK1/2/MAPK signaling pathway, the expression of mPGES-1 and EGR-1 was significantly reduced $(\mathrm{P}<0.05$; Fig. 8A), while the JNK and P38 inhibitors reduced the changes observed in EGR-1 and mPGES-1 (Fig. 8B and C). These results suggest that the ERK1/2/MAPK signaling pathway may regulate the expression of mPGES-1 through EGR-1.

\section{Discussion}

In the present study, the functional role of mPGES-1 silencing was elucidated in the proliferation, apoptosis and cell cycle of jurkat cells. Since it was first raised as a promising therapeutic target in 1999 (30), studies have revealed that prolonged and excessive production of mPGES-1 can alter a number of biological processes, leading to intractable pathologies, including inflammation and cancer (11-13). Therefore, blocking the expression of mPGES-1 is often an important strategy in treating these conditions. Previous studies by the authors have 

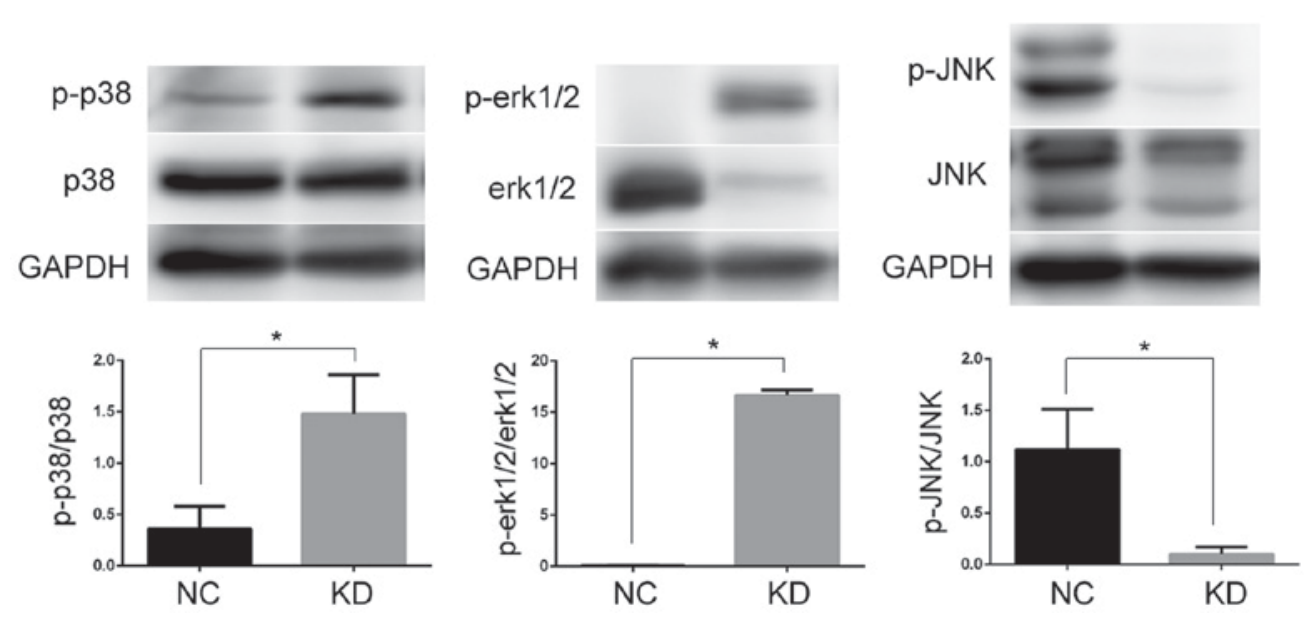

Figure 6. Effect of mPGES-1 silencing on MAPKs. Knockdown mPGES-1 significantly increased the phosphorylation of P38 and ERK1/2, while it decreased the phosphorylation level of JNK. "P<0.05. mPGES-1, microsomal prostaglandin E synthase-1; MAPK, mitogen-activated protein kinase; ERK1/2, extracellular signal regulated kinase; JNK, c-Jun N-terminal kinase; KD, jurkat cells transfected with shRNA (27743-1); NC, negative control.
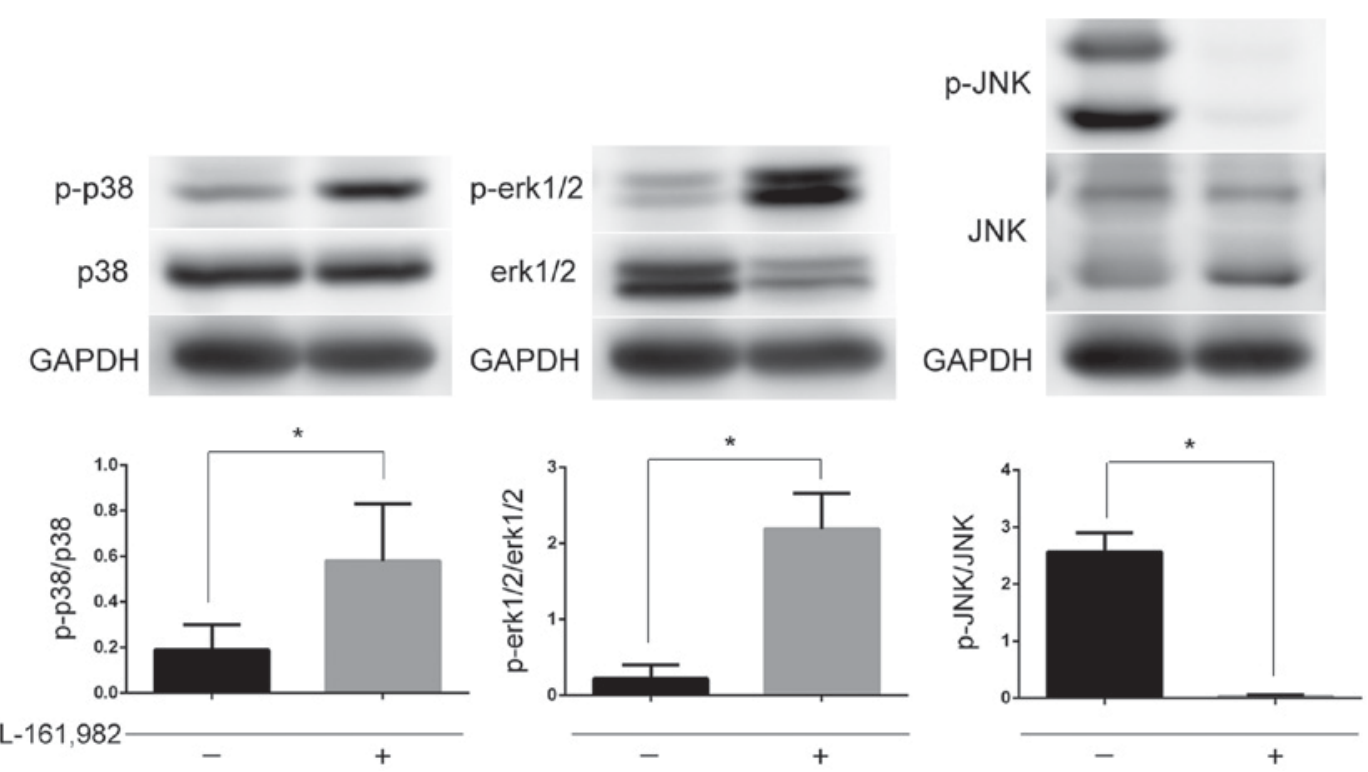

Figure 7. Effect of the EP4 receptor antagonist on MAPKs. Pre-incubating jurkat cells with the EP4 receptor antagonist L-161982 (20 $\mu$ mol/l) for $24 \mathrm{~h}$ increased the phosphorylation of P38 and ERK1/2, while it decreased the phosphorylation of JNK. "P $<0.05$. MAPK, mitogen-activated protein kinase; ERK1/2, extracellular signal regulated kinase; JNK, c-Jun N-terminal kinase; KD, jurkat cells transfected with shRNA (27743-1); NC, negative control.

confirmed that mPGES-1 is expressed highly in a variety of leukemia cells, including HL-60 (16), K562, jurkat and Raji (unpublished data). In the present study, it was revealed that decreasing mPGES-1 affected the growth of T-ALL jurkat cells and was also associated with the MAPK signaling pathway. As only one cell line was used in the experiments, it is not clear if such phenomenon exists in other T-ALL cell lines or primary cells; the authors believe that this is intriguing and worth exploring in future studies.

The roles of MAPKs in T-ALL have been previously described (31-33). The major MAPK signaling pathway subfamilies, including ERK1/2, JNK and P38 serve key roles in the regulation of the expression of various inflammatory genes, such as mPGES-1 (23). Based on these previous results, it was speculated that MAPKs may be involved in the regulation of mPGES-1 in T-ALL. Using microarray and western blot analysis, it was confirmed that mPGES-1 may affect the growth of jurkat cells via MAPKs. Interestingly, the effects of mPGES-1 on MAPK subfamilies were completely different. The JNK/MAPK signaling pathway was activated in the NC group, whereas this activation was inhibited in the KD group. However, the other two subfamilies, ERK1/2 and P38, displayed the opposite response following knockdown mPGES-1. These results indicated that mPGES-1 is located upstream of MAPKs, that MAPKs may be involved in the impact of mPGES-1 on jurkat cells and that the three subfamilies of MAPKs had different responses to mPGES-1 and there may be cross-talk between them; however, the exact mechanism requires further investigation.

mPGES-1 affects the function of tumor cells by increasing PGE2 synthesis. PGE2 exerts its biological actions by binding to four specific receptor subtypes known as EP1, EP2, EP3 and EP4 (34). The EP receptors are involved in the generation and progression of tumors through 

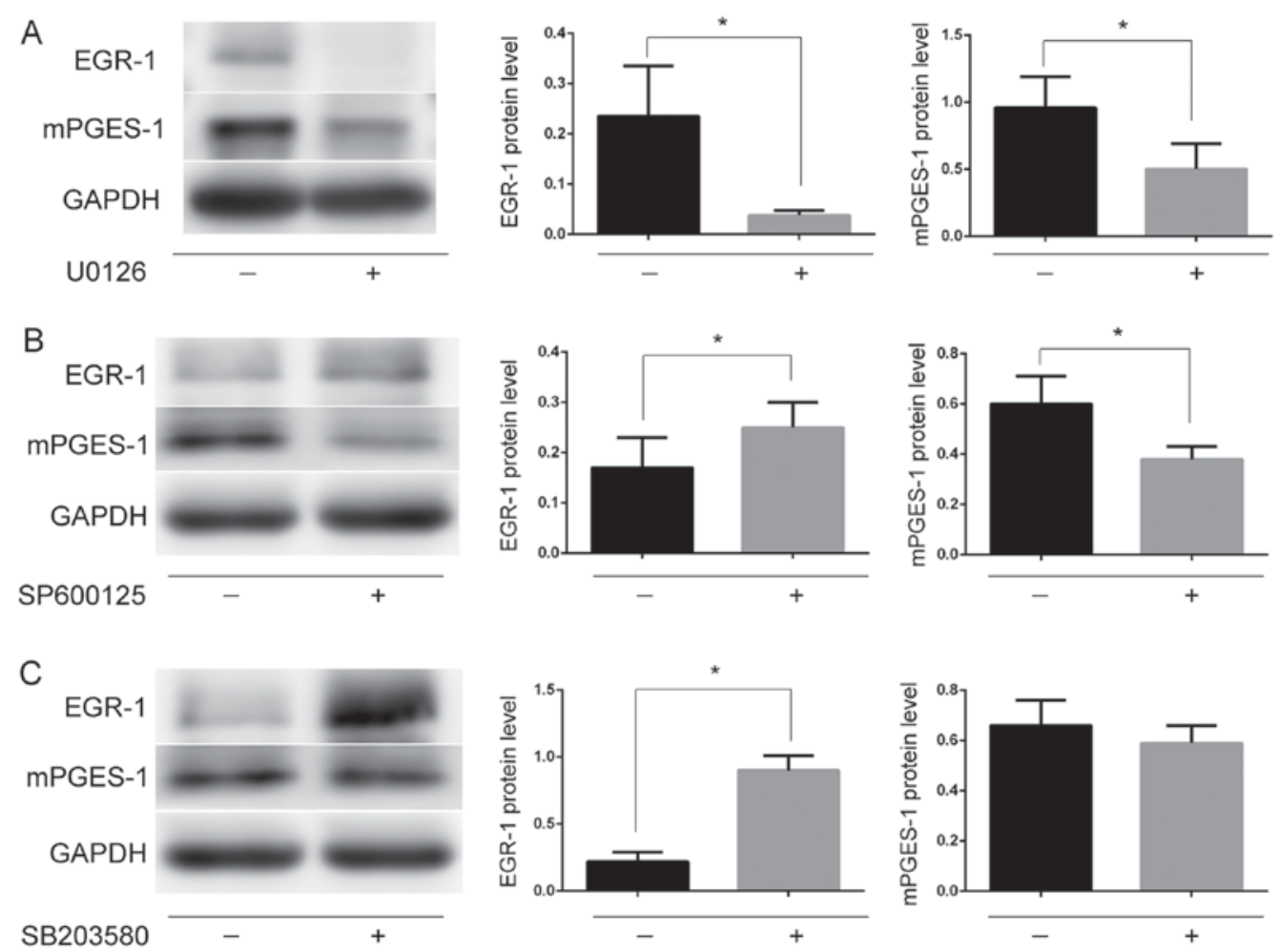

Figure 8. Effect of pre-incubating jurkat cells with MAPKs inhibitors on the expression of EGR-1 and mPGES-1. (A) Pre-incubated jurkat cells with MEK1/2 inhibitor U0126 ( $5 \mu \mathrm{mol} / 1$; for $2 \mathrm{~h}$ ) affected protein expression of EGR-1 and mPGES-1. (B) Pre-incubated jurkat cells with JNK inhibitor SP600125 (10 $\mu \mathrm{mol} / 1$; for $2 \mathrm{~h}$ ) affected protein expression of EGR-1 and mPGES-1. (C) Pre-incubated jurkat cells with P38 inhibitor SB203580 (10 $\mu$ mol/1; for 2 h) affected protein expression of EGR-1, but not mPGES-1. "P<0.05. mPGES-1, microsomal prostaglandin E synthase-1; MAPK, mitogen-activated protein kinase; ERK1/2, extracellular signal regulated kinase; JNK, c-Jun N-terminal kinase; EGR-1, early growth response protein-1.

the activation of different signaling pathways (35-37). Qian et al (38) reported that PGE2 stimulates human brain natriuretic peptide expression via the EP4-ERK1/2/MAPK signaling pathway. Mendez and LaPointe (39) also reported that PGE2 induces protein synthesis in cardiac myocytes, partly via activation of the EP4 receptor and subsequent activation of the ERK1/2/MAPK signaling pathway. In the present study the EP4 receptor was blocked by its antagonist, L-161982; this lead to a similar effect on MAPKs as those caused by mPGES-1 silencing. These results indicated that mPGES-1/PGE2 affected the growth of jurkat cells via EP4-dependent activation of the MAPK signaling pathway. However, the phosphorylated and activated subtype of MAPKs regulated by mPGES-1/PGE2/EP4 was different from that in Qian and Mendez's studies. This may be due to the different cell line used in the present experiment.

Accumulating evidence indicates that the activation of MAPKs is crucial for the expression of EGR-1 $(40,41)$. EGR-1 is a zinc finger transcription factor, which binds to GC-rich sequences, such as mPGES-1, in the regulatory region of its target genes (42). In the present study, blocking the ERK1/2/MAPK pathway induced a decrease in EGR-1 and mPGES-1, so it was speculated that the ERK1/2/MAPK signaling pathway may regulate mPGES-1 expression through EGR-1 (Fig. 9). Blocking the P38/MAPK or JNK/MAPK pathways induced an increase in EGR-1, while it decreased mPGES-1. These results may indicate the possibility of other potential mechanism underlying the regulation of mPGES-1, in addition to EGR-1.

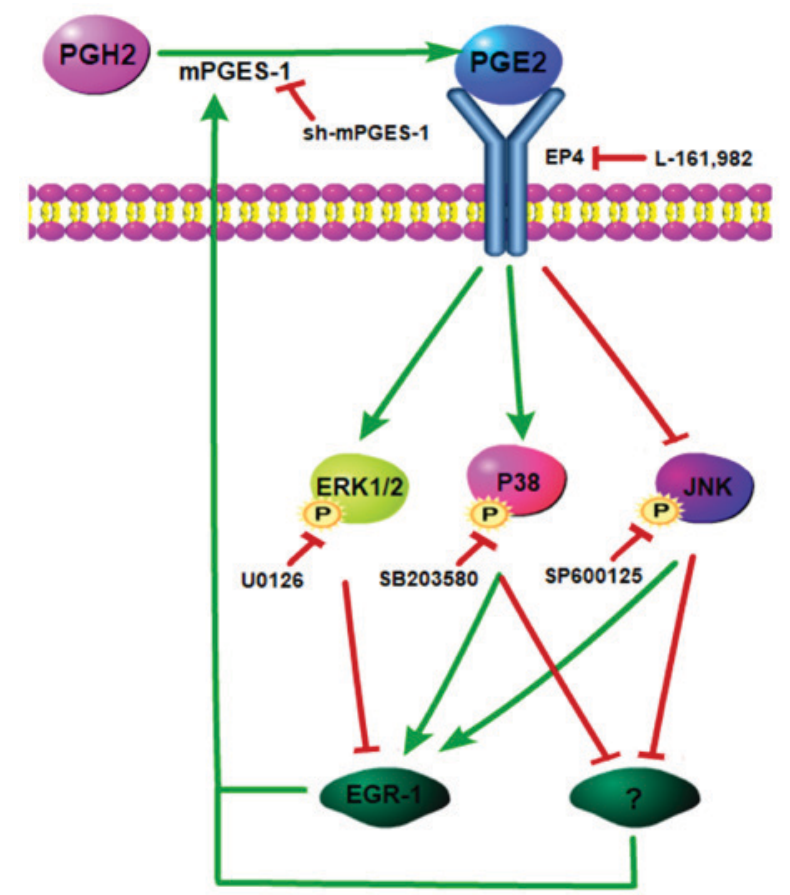

Figure 9. Model of the proposed association between mPGES-1 and MAPKs in jurkat cells. MAPK, mitogen-activated protein kinase; ERK1/2, extracellular signal regulated kinase; JNK, c-Jun N-terminal kinase; EGR-1, early growth response protein-1; PGE2, prostaglandin E2; $\mathrm{PGH} 2$, prostaglandin $\mathrm{H} 2$.

Based on the above findings, it is clear that mPGES-1 serves an important role in T-ALL jurkat cells by activating 
the JNK/MAPK signaling pathway, which in turn is required to achieve high levels of mPGES-1. This suggests that a positive feedback loop mediated by the JNK/MAPK signaling pathway promotes mPGES-1 induction. The results of the present study contradict the findings of a previous study, which mentioned a positive feedback loop between mPGES-1 and the ERK1/2/MAPK signaling pathway in macrophages (43). One potential explanation is that the present study performed experiments with all three of the classical subfamilies (ERK1/2/MAPK, JNK/MAPK and P38/MAPK) in jurkat cells, instead of macrophages. By exploring the growth of jurkat cells, it was observed that the effect of the positive feedback loop induced by the JNK/MAPK signaling pathway may be greater than that of the negative feedback induced by the ERK1/2/MAPK and P38/MAPK signaling pathways, subsequently leading to an inhibition of proliferation, induction of apoptosis and arrest of the cell cycle. However, the involvement of negative feedback loops may induce drug resistance or even treatment failure $(44,45)$. The findings of the present study raised the possibility that combined treatments of mPGES-1 with ERK1/2 and P38 inhibitors may be a novel therapeutic strategy for T-ALL.

\section{Acknowledgments}

Not applicable.

\section{Funding}

The present study was supported by grants from the National Natural Science Foundation of China (grant no. 81200342), the Guangdong Science and Technology Department (grant nos. 2014A020212085 and 2016A020215062), the Natural Science Foundation of Guangdong Province (grant no. 2016A030313360), the State Scholarship Fund of China (grant no. CSC 201606380189), the Key Laboratory of Malignant Tumor Molecular Mechanism and the Translational Medicine of Guangzhou Bureau of Science and Information Technology [(grant no. 163 (2013)] and the Key Laboratory of Malignant Tumor Gene Regulation and the Target Therapy of Guangdong Higher Education Institutes (grant no. KLB09001).

\section{Availability of data and materials}

The datasets used and/or analyzed during the current study are available from the corresponding author on reasonable request.

\section{Authors' contributions}

S-MY and D-NN designed the study, and analyzed and interpreted the data. Y-QL and J-TC conducted the experiments and contributed to writing the manuscript. All other authors, Z-YH, S-FX, X-JW, Y-DW, JX, H-YL, J-YW, W-JY and L-PM made substantial contributions to the experiments and the acquisition of data. All authors read and approved the final manuscript.

\section{Ethics approval and consent to participate}

Not applicable.

\section{Patient consent for publication}

Not applicable.

\section{Competing interests}

The authors declare that they have no competing interests.

\section{References}

1. Roti G and Stegmaier K: New approaches to target T-ALL. Front Oncol 4: 170, 2014.

2. Zhao WL: Targeted therapy in T-cell malignancies: dysregulation of the cellular signaling pathways. Leukemia 24: 13-21, 2010

3. Qiu X, Cheng JC, Chang HM and Leung PC: COX2 and PGE2 mediate EGF-induced E-cadherin-independent human ovarian cancer cell invasion. Endocr Relat Cancer 21: 533-543, 2014.

4. Pan J, Cheng L, Bi X, Zhang X, Liu S, Bai X, Li F and Zhao AZ: Elevation of $\mathrm{w}-3$ polyunsaturated fatty acids attenuates PTEN-deficiency induced endometrial cancer development through regulation of COX-2 and PGE2 production. Sci Rep 5: 14958, 2015.

5. Larsson K, Kock A, Idborg H, Arsenian Henriksson M, Martinsson T, Johnsen JI, Korotkova M, Kogner P and Jakobsson PJ: COX/mPGES-1/PGE2 pathway depicts an inflammatory-dependent high-risk neuroblastoma subset. Proc Natl Acad Sci U S A 112: 8070-8075, 2015.

6. Steinbach G, Lynch PM, Phillips RK, Wallace MH, Hawk E, Gordon GB, Wakabayashi N, Saunders B, Shen Y, Fujimura T, et al: The effect of celecoxib, a cyclooxygenase-2 inhibitor, in familial adenomatous polyposis. N Engl J Med 342: 1946-1952, 2000.

7. Howe LR and Dannenberg AJ: COX-2 inhibitors for the prevention of breast cancer. J Mammary Gland Biol Neoplasia 8: 31-43, 2003.

8. Yiannakopoulou E: Aspirin and NSAIDs for breast cancer chemoprevention. Eur J Cancer Prev 24: 416-421, 2015.

9. Fosbol EL, Kober L, Torp-Pedersen C and Gislason GH: Cardiovascular safety of non-steroidal anti-inflammatory drugs among healthy individuals. Expert Opin Drug Saf 9: 893-903, 2010.

10. Takeuchi K: Pathogenesis of NSAID-induced gastric damage: Importance of cyclooxygenase inhibition and gastric hypermotility. World J Gastroenterol 18: 2147-2160, 2012.

11. Larsson $\mathrm{K}$ and Jakobsson PJ: Inhibition of microsomal prostaglandin E synthase-1 as targeted therapy in cancer treatment. Prostaglandins Other Lipid Mediat 120: 161-165, 2015.

12. Olesch C, Sha W, Angioni C, Sha LK, Açaf E, Patrignani P, Jakobsson PJ, Radeke HH, Grösch S, Geisslinger G, et al: MPGES-1-derived PGE2 suppresses CD80 expression on tumor-associated phagocytes to inhibit anti-tumor immune responses in breast cancer. Oncotarget 6: 10284-10296, 2015.

13. Finetti F, Terzuoli E, Giachetti A, Santi R, Villari D, Hanaka H, Radmark O, Ziche $M$ and Donnini S: mPGES-1 in prostate cancer controls stemness and amplifies epidermal growth factor receptor-driven oncogenicity. Endocr Relat Cancer 22: 665-678, 2015.

14. Li YQ, Yin SM, Xie SF, Wang XJ, Ma LP, Nie DN and Wu YD: Effect of mPGES-1 inhibitor MK886 on cell cycle of leukemia HL-60 cells. Zhongguo Shi Yan Xue Ye Xue Za Zhi 20: 1072-1076, 2012 (In Chinese).

15. Li YQ, Yin SM, Nie DN, Xie SF, Ma LP, Wang XJ and Wu YD: Effect of mPGES-1 inhibitor MK886 on apoptosis and drug resistance of HL-60/A cells. Zhongguo Shi Yan Xue Ye Xue Za Zhi 20: 829-834, 2012 (In Chinese).

16. Li Y, Yin S, Nie D, Xie S, Ma L, Wang X, Wu Y and Xiao J: MK886 inhibits the proliferation of HL-60 leukemia cells by suppressing the expression of mPGES-1 and reducing prostaglandin E2 synthesis. Int J Hematol 94: 472-478, 2011.

17. Munoz L and Ammit AJ: Targeting p38 MAPK pathway for the treatment of Alzheimer's disease. Neuropharmacology 58: 561-568, 2010.

18. Olajide OA, Bhatia HS, de Oliveira AC, Wright CW and Fiebich BL: Anti-neuroinflammatory properties of synthetic cryptolepine in human neuroblastoma cells: Possible involvement of NF- $\kappa$ B and p38 MAPK inhibition. Eur J Med Chem 63: 333-339, 2013. 
19. Furuya H, Wada M, Shimizu Y, Yamada PM, Hannun YA Obeid LM and Kawamori T: Effect of sphingosine kinase 1 inhibition on blood pressure. FASEB J 27: 656-664, 2013.

20. Olajide OA, Velagapudi R, Okorji UP, Sarker SD and Fiebich BL: Picralima nitida seeds suppress PGE2 production by interfering with multiple signalling pathways in IL-1 $\beta$-stimulated SK-N-SH neuronal cells. J Ethnopharmacol 152: 377-383, 2014.

21. Okorji UP, Velagapudi R, El-Bakoush A, Fiebich BL and Olajide OA: Antimalarial drug artemether inhibits neuroinflammation in BV2 microglia through Nrf2-dependent mechanisms Mol Neurobiol 53: 6426-6443, 2016.

22. Hu H, Goltsov A, Bown JL, Sims AH, Langdon SP, Harrison DJ and Faratian D: Feedforward and feedback regulation of the MAPK and PI3K oscillatory circuit in breast cancer. Cell Signal 25: 26-32, 2013.

23. Bhatia HS, Baron J, Hagl S, Eckert GP and Fiebich BL: Rice bran derivatives alleviate microglia activation: Possible involvement of MAPK pathway. J Neuroinflammation 13: 148, 2016.

24. Fu P, Liang GJ, Khot SS, Phan R and Bach LA: Cross-talk between MAP kinase pathways is involved in IGF-independent, IGFBP-6-induced Rh30 rhabdomyosarcoma cell migration. J Cell Physiol 224: 636-643, 2010.

25. O'Callaghan G and Houston A: Prostaglandin E2 and the EP receptors in malignancy: Possible therapeutic targets? Br J Pharmacol 172: 5239-5250, 2015.

26. Diaz-Munoz MD, Osma-Garcia IC, Cacheiro-Llaguno C, Fresno $\mathrm{M}$ and Iniguez MA: Coordinated up-regulation of cyclooxygenase-2 and microsomal prostaglandin E synthase 1 transcription by nuclear factor kappa B and early growth response-1 in macrophages. Cell Signal 22: 1427-1436, 2010.

27. Chabane N, Li X and Fahmi H: HDAC4 contributes to IL-1-induced mPGES-1 expression in human synovial fibroblasts through up-regulation of Egr-1 transcriptional activity. J Cell Biochem 106: 453-463, 2009.

28. Moon Y, Lee M and Yang H: Involvement of early growth response gene 1 in the modulation of microsomal prostaglandin E synthase 1 by epigallocatechin gallate in A549 human pulmonary epithelial cells. Biochem Pharmacol 73: 125-135, 2007.

29. Noma T, Takahashi-Yanaga F, Arioka M, Mori Y and Sasaguri T: Inhibition of GSK-3 reduces prostaglandin E2 production by decreasing the expression levels of COX-2 and mPGES-1 in monocyte/macrophage lineage cells. Biochem Pharmacol 116: $120-129,2016$

30. Jakobsson PJ, Thoren S, Morgenstern R and Samuelsson B: Identification of human prostaglandin E synthase: A microsomal, glutathione-dependent, inducible enzyme, constituting a potential novel drug target. Proc Natl Acad Sci U S A 96: 7220-7225, 1999.

31. Tomiyasu H, Watanabe M, Sugita K, Goto-Koshino Y, Fujino Y, Ohno K, Sugano S and Tsujimoto H: Regulations of ABCB1 and ABCG2 expression through MAPK pathways in acute lymphoblastic leukemia cell lines. Anticancer Res 33: 5317-5323, 2013.

32. Liu Y, Ge J, Li Q, Guo X, Gu L, Ma ZG, Li XH and Zhu YP: Low-dose anisomycin sensitizes glucocorticoid-resistant T-acute lymphoblastic leukemia CEM-Cl cells to dexamethasone-induced apoptosis through activation of glucocorticoid receptor and p38-MAPK/JNK. Leuk Lymphoma 55: 2179-2188, 2014.
33. Naci D and Aoudjit F: Alpha2beta1 integrin promotes T cell survival and migration through the concomitant activation of ERK/Mcl-1 and p38 MAPK pathways. Cell Signal 26: 2008-2015, 2014.

34. Kawahara K, Hohjoh H, Inazumi T, Tsuchiya S and Sugimoto Y: Prostaglandin E2-induced inflammation: Relevance of prostaglandin E receptors. Biochim Biophys Acta 1851: 414-421, 2015

35. Xia S, Ma J, Bai X, Zhang H, Cheng S, Zhang M, Zhang L, Du M, Wang Y, Li H, et al: Prostaglandin E2 promotes the cell growth and invasive ability of hepatocellular carcinoma cells by upregulating c-Myc expression via EP4 receptor and the PKA signaling pathway. Oncol Rep 32: 1521-1530, 2014.

36. Du M, Shi F, Zhang H, Xia S, Zhang M, Ma J, Bai X, Zhang L, Wang Y, Cheng S, et al: Prostaglandin E2 promotes human cholangiocarcinoma cell proliferation, migration and invasion through the upregulation of $\beta$-catenin expression via EP3-4 receptor. Oncol Rep 34: 715-726, 2015.

37. Kim KM, Im AR, Kim SH, Hyun JW and Chae S: Timosaponin AIII inhibits melanoma cell migration by suppressing COX-2 and in vivo tumor metastasis. Cancer Sci 107: 181-188, 2016.

38. Qian JY, Leung A, Harding P and LaPointe MC: PGE2 stimulates human brain natriuretic peptide expression via EP4 and p42/44 MAPK. Am J Physiol Heart Circ Physiol 290: H1740-H1746, 2006.

39. Mendez M and LaPointe MC: PGE2-induced hypertrophy of cardiac myocytes involves EP4 receptor-dependent activation of p42/44 MAPK and EGFR transactivation. Am J Physiol Heart Circ Physiol 288: H2111-2117, 2005.

40. Ryu WI, Lee H, Kim JH, Bae HC, Ryu HJ and Son SW: IL-33 induces Egr-1-dependent TSLP expression via the MAPK pathways in human keratinocytes. Exp Dermatol 24: 857-863, 2015.

41. Jeong SH, Kim HJ, Ryu HJ, Ryu WI, Park YH, Bae HC, Jang YS and Son SW: ZnO nanoparticles induce TNF- $\alpha$ expression via ROS-ERK-Egr-1 pathway in human keratinocytes. J Dermatol Sci 72: 263-273, 2013

42. Naraba H, Yokoyama C, Tago N, Murakami M, Kudo I, Fueki M, Oh-Ishi S and Tanabe T: Transcriptional regulation of the membrane-associated prostaglandin E2 synthase gene. Essential role of the transcription factor Egr-1. J Biol Chem 277: 28601-28608, 2002.

43. Khan KM, Kothari P, Du B, Dannenberg AJ and Falcone DJ: Matrix metalloproteinase-dependent microsomal prostaglandin E synthase-1 expression in macrophages: Role of TNF- $\alpha$ and the EP4 prostanoid receptor. J Immunol 188: 1970-1980, 2012.

44. Galoian K, Temple HT and Galoyan A: mTORC1 inhibition and ECM-cell adhesion-independent drug resistance via PI3K-AKT and PI3K-RAS-MAPK feedback loops. Tumor Biol 33: 885-890, 2012.

45. Mirzoeva OK, Das D, Heiser LM, Bhattacharya S, Siwak D, Gendelman R, Bayani N, Wang NJ, Neve RM, Guan Y, et al: Basal subtype and MAPK/ERK kinase (MEK)-phosphoinositide 3-kinase feedback signaling determine susceptibility of breast cancer cells to MEK inhibition. Cancer Res 69: 565-572, 2009. 